\title{
Design of a Wireless System for Patient-Hospital Communication and Result Validation in Point of Care Testing
}

John McGrory

Technological University Dublin, john.mcgrory@tudublin.ie

Owen Lynch

Technological University Dublin, owen.lynch@tudublin.ie

Eugene Coyle

Technological University Dublin, Eugene.Coyle@tudublin.ie

Follow this and additional works at: https://arrow.tudublin.ie/teapotcon

Part of the Computer and Systems Architecture Commons, and the Medicine and Health Sciences

Commons

\section{Recommended Citation}

McGrory,J.,Lynch,O., Coyle,E:Design of a Wireless System for Patient-Hospital Communication and Result Validation in Point of Care Testing. ICCIMA'05: the International Conference of Computational Intelligence and Multimedia Applications University of Nevada, Las Vegas,USA.

This Conference Paper is brought to you for free and open access by the tPOT: People Oriented Technology at ARROW@TU Dublin. It has been accepted for inclusion in Conference Papers by an authorized administrator of ARROW@TU Dublin. For more information, please contact arrow.admin@tudublin.ie, aisling.coyne@tudublin.ie,gerard.connolly@tudublin.ie. Funder: Technological University Dublin, Masters Research 


\title{
Design of a Wireless System for Patient-Hospital Communication and Result Validation in Point of Care Testing
}

\author{
John McGrory, Owen Lynch and Eugene Coyle \\ Dublin Institute of Technology, Ireland \\ john.mcgrory@dit.ie,owen.lynch@dit.ie andEugene.coyle@dit.ie \\ http://www.dit.ie
}

\begin{abstract}
This paper discuses mobile phone (cell phone) and wireless applications for linking patients who manage their healthcare outside the hospital using Point of Care Testing (POCT) to hospital information systems (HIS). Certain medical conditions require patients to manage their healthcare by performing on themselves POC testing and act faithfully on the result. This raises quality control issue, as these POC samples and testing procedures are not independently overseen by professional hospital staff. In hospitals, samples taken by clinicians are validated by hi-tech computerised validation systems to ensure plausibility, before physicians rely on them. Patients in the home must often use results from these POCT to determine medication dosage or to monitor their condition. Thus, there is a need to implement a system of result validation, either locally or by the hospital validation system itself, for people testing with POCT devices.
\end{abstract}

\section{Introduction}

\subsection{Point of care testing}

POCT gives patients an opportunity to manage their own conditions, reduce hospital stays and minimise related costs associated with hospital testing for example, patient time and travel. With an ageing worldwide population, set to double by 2025 [1], there is a need to increase the use of POCT in the healthcare system model. POCT to homebound patients could have significant impact on their condition [2]. The diversity of POCT apparatus available is as varied as the ailments themselves ranging from simple urine dipsticks and blood pressure arm cuffs to complicated ECG devices. Patients also use blood-testing units, such as glucometers for diabetics to check glucose levels or blood coagulation (INR) meters for people taking anticoagulant medication, such as warfarin. The management of diabetes in the USA costs $\$ 100$ billion annually and has many secondary disorders associated with it, however management of the condition with POCT would minimise many of these disorders [2].

\subsection{Clinical testing and result validation}

For a physician to manage an illness it is common practice to gather patient samples for analysis (blood, urine, faeces etc.). The clinical laboratory applies gold standard testing regimes on each sample to obtain a result. The gold standard is considered the most specific and sensitive test for this sample type and completed strictly according to a workflow list. Once a result is obtained the laboratory begins checking if this result is plausible for this patient in this instance. Result validation is generally divided into two areas, namely "Technical validation" and "Clinical validation".

Technical validation checks for instrument calibration, sample tolerance, repeatability, reliability and certification. This insures the results are from a calibrated, certified process, the results are consistent and samples are not contaminated. Clinical validation on the other hand is when the result is checked for plausibility. They ensure results are relevant to the patient and the complaint being investigated. 
Table 1 illustrates general tests undertaken by a clinical laboratory in the validation of a sample, specifically blood.

\begin{tabular}{|l|l|}
\hline Type of validation checks & Description \\
\hline Calibration & Instrument checked is calibrated and working correctly. \\
\hline Instrument specific checks & $\begin{array}{l}\text { Vary depending on instrument and analytical process used } \\
\text { to generate the result. Often carried out either by the } \\
\text { instrument itself or manually by the instrument operator. }\end{array}$ \\
\hline Internal consistency checks & $\begin{array}{l}\text { The consistency between pathophysiologically related } \\
\text { variables is examined }\end{array}$ \\
\hline Basic validation checks & $\begin{array}{l}\text { Data checked against age and sex related reference ranges, } \\
\text { pathological limits as set down by the laboratory based on } \\
\text { international standards. }\end{array}$ \\
\hline Delta checks & $\begin{array}{l}\text { Previous results are compared with the current results } \\
\text { using various techniques. }\end{array}$ \\
\hline Sample mix-ups & $\begin{array}{l}\text { When one sample is given the identity of another, usually } \\
\text { an adjacent sample on the workbench }\end{array}$ \\
\hline
\end{tabular}

Table 1, Advanced Validation Checks [4] Modified

When the checks are completed and if the sample results are still plausible the data is considered validated and populated into the patient's healthcare record. If at any stage the results are not plausible then the patient information is forwarded to the laboratory manager were a retest of the sample can be authorized or a new sample requested. POCT should be validated in a similar fashion where possible to the clinical laboratory test for consistency.

\subsection{Home testing issues}

For patients who perform tests at home with POCT equipment, there are four major issues which this research addresses.

(i) Testing quality achieved by patients operating self monitoring instruments is lesser when compared to a technician using the same instrument [11].

(ii) No independent validation of the test results. Thus decisions made as a consequence of POCT may be erroneous, potentially leading to patient complications or death.

(iii) The hospital professionals and doctors are unaware of patient progress between hospital visits. Complications arises between visits, it may go unnoticed.

(iv) Prescribed drugs and diet interacting and affecting test results. For example, large amounts of broccoli, spinach, and other green leafy vegetables high in vitamin $\mathrm{K}$ promote the formation of blood clots counteract the effects of warfarin, and other drugs given to prevent clotting.

Hospital validation servers made aware of these can change validation parameters or validation algorithm, tuning its accuracy and making comments useful to the patient.

\subsection{Proposed solution}

A major challenge of POCT is the integration of test results into hospital information systems (HIS) and electronic patient records (EPR). The need for connectivity between POCT devices and HIS is well recognised and benefits have been documented [5]. This research endeavours to bridge the gap between the patient at home and the hospital responsible for the treatment of the patient. Some solutions are proposed for connecting patients with the hospital system for validation of results and the population of data to their EPR, using a mobile phone. 


\section{Mobile phone and java technologies}

\subsection{Introduction}

With the worldwide mobile phone subscribers passing 1.5 billion in 2004 and predicted to reach 2 billion by July 2006 [6], it is clear mobile phones are a familiar tool and are becoming a necessary tool in everyday life for many people. Phones are now more than just simple voice communicators and are evolving into sophisticated mini-computers, capable of running small to medium sized applications. Research has been carried out to investigate different mobile technologies that could be used in the solution for validating results of patients using POCT equipment.

\subsection{Relevant mobile phone technologies}

Three of the technologies considered for use as a possible solution were Short Message Service (SMS), Wireless Application Protocol (WAP) and Java 2 Micro Edition (J2ME).

SMS is a short text-based message for simple communication between phone subscribers, but a stream of SMS messages can be used to transmit any digital data. However, SMS is a store-and-forward service and there is no guarantee that the information will be delivered in a timely manner [7]. As POCT is time critical, this would not be acceptable.

WAP is an open specification allowing wireless devices easily interact with services and permits users to access the Internet [8]. For example, patients accessing the hospital server submit test results and have relevant information returned to them, both textually and graphically. It uses the GPRS network, which is an "always-on" service and provides the highest possible transmission rates in GSM networks. However, there are also limitations in using WAP as a solution. It requires a constant connection to the network and thus an off-line solution for validation would not be possible.

J2ME is lighter than standard Java designed specifically for developing applications on wireless communications devices with limited memory sizes, such as mobile telephones. There are hundreds of phones capable of running J2ME [9] and it is becoming a standard feature on mobile phones. J2ME applications are developed as MIDlets, MIDlets are to wireless what Java Applets have been to the web. J2ME applications can run on the phone without a network connection and are capable of making HTTP connections to Internet servers and parsing the response. They are capable of storing data persistently, displaying data graphically and can perform relatively complex computation. J2ME has the ability to handle and parse XML, a format for structured documents and data that facilitates the interchange of data between computer applications. Explorative work on simple agents has allowed the automated selection of the most suitable hospital service server available from a range of services for the sample result to be validated using XML. Therefore, J2ME has been chosen to develop the applications for ensuring high quality result validation.

\subsection{Java servlets}

J2ME applications can wrap any data into an XML document and send it to a server. Java Servlet technology operates using this technique. It provides a mechanism for extending the functionality of a web server and accessing business systems [10]. Java servlets have access to all of the existing Java application programming interfaces (APIs), so that powerful server programs can be accessed by simple client applications remotely. Java Servlets have been chosen to implement the test hospital server programs and will be used for validating the results [12], providing software updates for the client application and storing the patient results in the EPR. 


\section{System design}

\subsection{Introduction}

Three versions of the application have been considered. All applications consist of a J2ME client application, running on the phone, and server application implemented as a Java Servlet. For simplicity we have omitted the pre-test instruction/procedure sets which are necessary irrespective of version chosen. In the first version, the phone application performs the validation of the result, only connecting to the hospital to update the validation procedure, and upload the result to the hospital database. In the second version the hospital server processes all validation computation, making the mobile client application much smaller. The third version divides the workload between the phone and server, so some initial computation may be done on the phone, but the application can remotely call procedures on the server for more heavy computation and uploading of result data.

\subsection{Version 1: All computation on the phone}

The client application runs the rule base and algorithm to validate the patient's result locally. The rules could be tailored specifically for a patient. This method is suitable for result types where the validation procedure is relatively simple. PDA and mobile phone processor speeds vary between $200-550 \mathrm{MHz}$, with cache memory and bus speeds characteristics clearly restricting their processing capability. In addition programmers have no homogeneous access to lower level apparatus, for example camera or battery power remaining features on the phone without resorting to vendor/phone specific java class sets. This restricts the development of the "all on phone" processing capability. Once the validation is completed, the application connects to the hospital and transmits the result data in HL7 XML format [12] for storage on the hospital database. A simple workflow of the system is shown in Figure 2. Previous results will be stored locally on the phone, but can be retrieved from the hospital database also. The validation algorithm may be updated and changed by the hospital technicians at any time; the application designed allows for this with functionality to check for an update. If one is available, it downloads the new data and uses it for validation of results. Although all three versions of the application will need to make network connections, this one will generally use the least "air-time" as it does not need to wait for a response from the hospital validation system to validate the results.

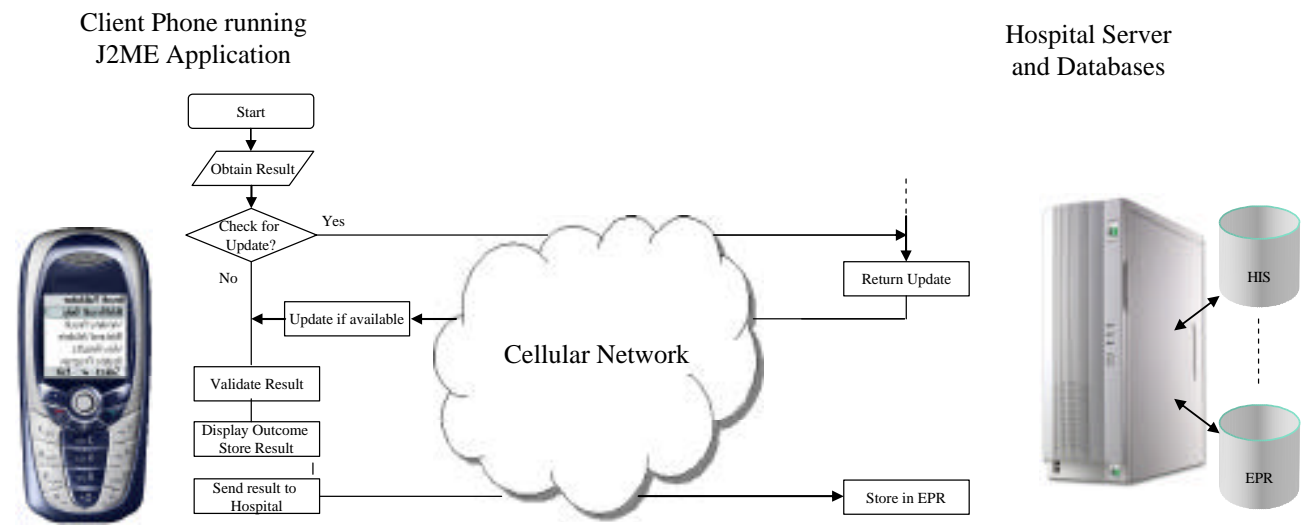

Figure 1: Simplified workflow for the first version of the system.

\subsection{Version 2: All computation on the server}

This application minimises the work done by the client application on the actual validation of the result. Information entered by the user on the phone is wrapped in an XML document and sent to the server over the HTTP connection. The result is then validated on the server by the validation process. If the result is valid it is stored on the patients EPR. If invalid, it is marked for inspection by the relevant hospital staff. A response for the client is generated and 
the outcome of the validation is included in it. Figure 2 depicts a simplified workflow of how the client and servers would work together in the system. This client application needs to connect the patient's result with the hospital server ensuring results are validated by the same quality validation service as the one validating results of hospital tests.

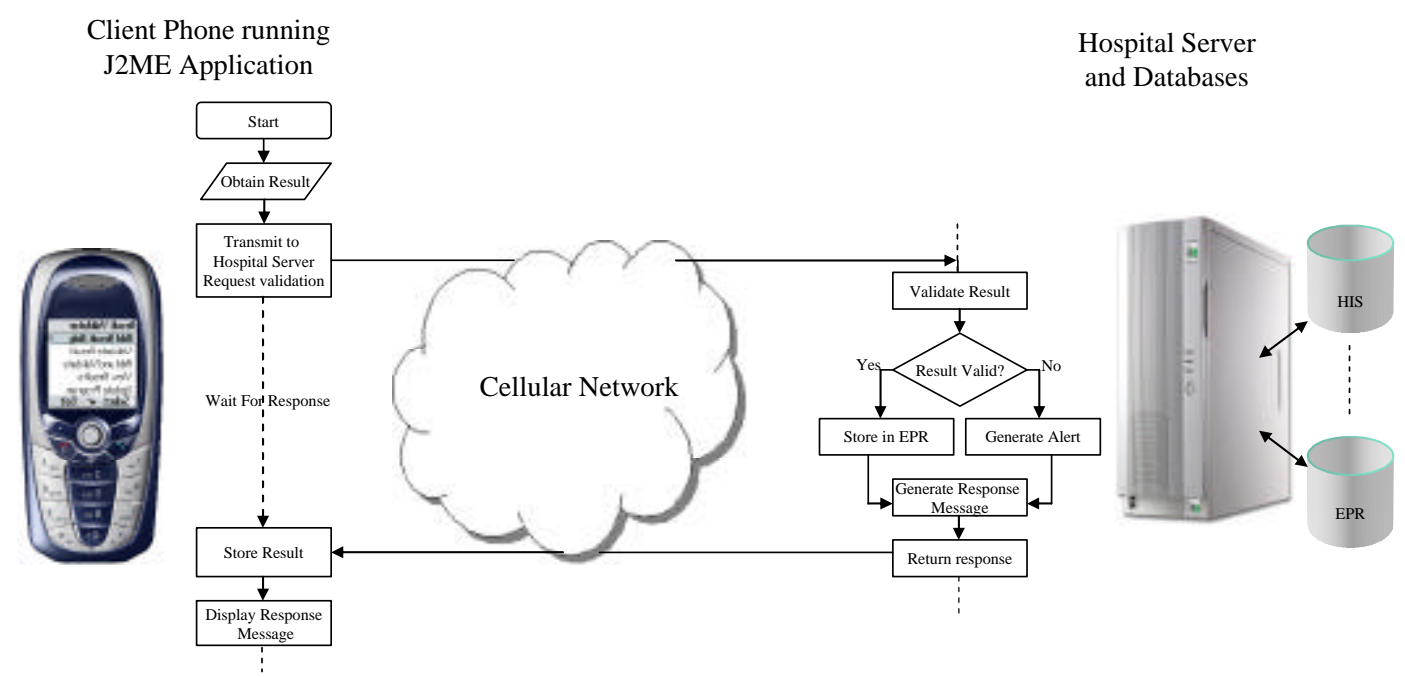

Figure 2: Simplified workflow for the second version of the system.

\subsection{Version 3: Shared computation}

This version could potentially be the most useful/appropriate application and is similar to the second version described above. When results to be validated enter this application they invoke the method for validation on the server remotely. This could be achieved using mobile agents. The agent architecture brokers communications with the most suitable hospital validation service. To the user it will seem like the application is doing the computation locally but in fact it will be done by the server application. This distributed solution means the client application can be smaller than the first version, which is more suitable for a J2ME device. It also means, as in the previous version, the result is being validated by the hospital validation service, which will always be the most up to date version. XML-RPC is a lightweight XML-based protocol for remote method invocation over HTTP and is being considered for use in this version. Figure 2 shows a simplified workflow for this third version.

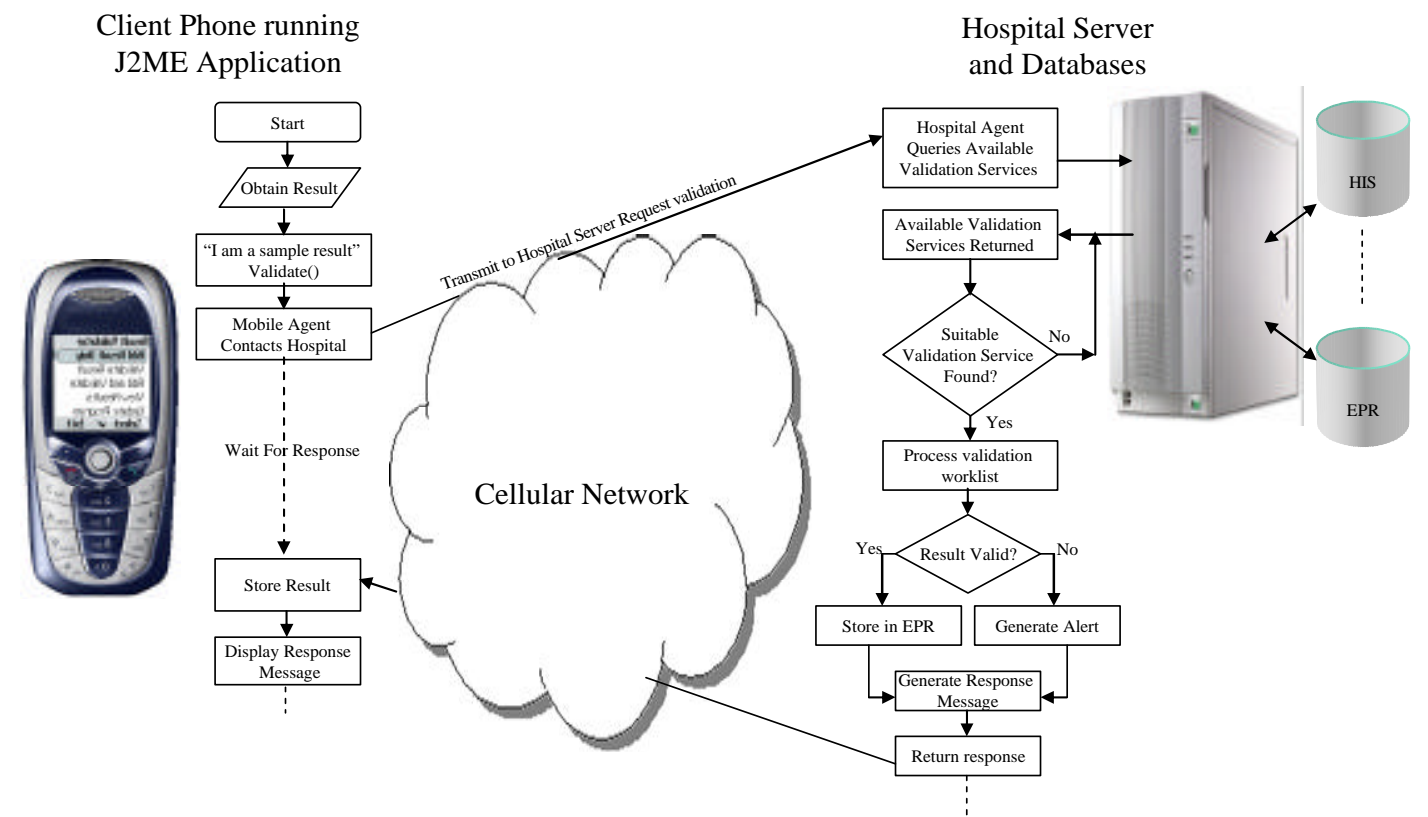

Figure 3: Simplified workflow for the third version. 


\section{Planned implementation}

\subsection{Building and testing the system}

The basic designs for the three versions of the system have now been outlined. The implementations for both the client and server applications are being carried out and the selection of the optimal design from the three versions described will be based on the results of comprehensive testing and benchmarking of the systems. There are good reasons for implementing each version by testing each one and comparing the results of all three will confirm which ones are viable solutions. Factors being considered to determine the optimum system will be; the accuracy of the result validation compared to the gold standard, processing time, scalability and network traffic efficiency.

\subsection{Application deployment}

For all three cases the method for deploying the application will be the same. J2ME applications can be deployed to mobile phones over the air (OTA) and thus users are not required to have data cables for their handsets. The process will work as follows; the patient downloads the application to their phone from the hospital server. The application runs locally but can make HTTP connection to the hospital to upload data or validate the result. The client application receives data back and can perform additional computation if necessary and store the result locally.

\section{Conclusion}

It has been shown that with the increase in the demand for POCT in the home there is a great opportunity for work in the area of patient-hospital communication. The applications outlined in this paper give a guideline as to how such a system may be realised. With the roll out of $3 \mathrm{G}$ networks already in operation there is great scope for expanding these applications, thus ensuring greater patient care and less time wasted. With the increased volume of information now being gained from POCT there is an increased demand on when, where and how the information is obtained and stored. Agent brokering theory is being used to improve the selection and execution difficulties experienced with distributed technologies [13].

\section{References}

[1] World Health Organization, http://www.who.int/hpr/ageing/ageing.pdf accessed March 2005.

[2] Lehmann, C. A., "The Future of Home Testing - implications for traditional laboratories", Clinica Chimica Acta, Vol 323 pages 21 - 36, September 2002.

[3] Oosterhuis W. P., Herman J. L. M., Goldschmidt H. M. J., "Evaluation of LabRespond, a new Automated Validation System for Clinical Laboratory Test Results", Clinical Chemistry Volume 46, Issue 11, November 2000

[4] Boran G., Given P., O’Moore R., "Patient Result Validation Services, Computer Methods and Programs in Biomedicine", Vol 50 Page 161 - 168, 1996.

[5] Taylor, M., Nichols, J.H., Saltz, J., "POCT Connectivity, Opening the door to a laboratory without walls", American Clinical Laboratory, July 2000.

[6] Josifovska S., "Where Next For The Handset?", IEE Review Volume 50, Number 12, December 2004.

[7] Knyziak T., Winiecki W., "The new prospects of distributed measurement systems using Java 2 Micro Edition mobile phone", Computer Standards and Interfaces, Article in press, available online February 2005 at www.sciencedirect.com

[8] Read K., Maurer F., "Developing Mobile Wireless Applications", IEEE Internet Computing, January/February 2003, http://computer.org/internet/

[9] Java 2 Platform, Micro Edition (J2ME), http://java.sun.com/j2me/

[10] Java Servlet Technology, http://java.sun.com/products/servlet/

[11] Skeie Svein, Geir Thue, Kari Nerhus and Sverre Sandberg "Instruments for Self-Monitoring of Blood Glucose: Comparisons of Testing Quality Achieved by Patients and a Technician" published by Clin. Chem., Jul 2002; 48: 994 - 1003.

[12] HL7 messaging system schema, http://www.hl7.org, accessed Dec 04

[13] Agent principles, http://agent.omg.org, accessed Dec 04. 